\title{
RATIONAL CHOICE FUNCTION DERIVED FROM A FUZZY PREFERENCE
}

\author{
JIN BAI KIM \\ Departinent of Mathematics \\ west lirginia iniversity \\ Morgantown, W. V. 26506 \\ KERN O. KYMN \\ Department of Economics \\ West Virginia liniversity \\ Morgantown, W. V. 26506 \\ (Received September 7, 1984 and in revised form April 22, 1987)
}

ABSTRACT. We shall prove that every fuzzy rational choice function is fuzzy regular (see Richter $16, p .36 \mathrm{~J})$, count the total number of the fuzzy rational cholce functions on a set of four elements and consider a semigroup of all fuzzy rational choice functions on a set.

KEY WORDS AND PHRASES. Fuzzy relation - fuzzy binary relation - fuzzy preference - choice function - fuzzy rational choice function - fuzzy transitive - fuzzy regular - semigroup. 1985 AMS CLASSIFICATION NIMBER 03E72

1. INTRODUCTION. We have introduced a rational choice function derived from a fuzzy preference (see $[2],[3],[4])$. We shall establish two theorems (Theorems 1 and 2 ) which are motivated from the following theorems:

THEOREM 4 (Richter [6]). There exists a total rational choice which is not transitive rational.

THEOREM 6 (Richter [6]). There exists a rational choice which is not total rational.

We $f$ ind that the number of all fuzzy rational choice functions on a set $x=\{a, b$, c, d) of four elements is equal to 57751 (see [2]. We shall consider a semigroup. We note that in [4] there is a beautiful counting formula of the total number of all final choice functions on a finite set.

2. DEFINITIONS AND THEOREMS.

Let $\mathrm{X}$ be a finite set with more than two elements. For definitions of a choice function on $X$ and a fuzzy binary relation $(R, r)$ on $X$, we refer to [2] and [3].

DEFINITION 1 [2, p. 38$\}$. Let $(R, r)$ be a fuzzy relation $X$ and let $a \varepsilon X$. Define $R(a)=\left\{\begin{array}{llll}x & \varepsilon & X & \text { : }\end{array}\right.$ Rx and $\left.r(a, x) \neq 0\right\}$ and $R_{t}(a)=\left\{x \in R(a): r(a, x) \geqq \frac{1}{t}\right\}$ for $\left.\frac{1}{t} \varepsilon \quad(0,1)\right]$. We define a function $h_{R}$ as follows: Let $a \varepsilon A \subseteq X$. Then $a_{\varepsilon}$ $h_{R}(A)$ iff $A \subseteq R_{A}(a)$. We add that $h_{R}(\emptyset)=\varnothing$, the empty set. Note that $h_{R}$ is in general, not a choice function. Let $h$ be a choice function on $X$. If there exists a fuzzy relation $(R, r)$ on $X$ such that $h_{R}=h$, then we shall say that $h$ is 
fuzis rational and $(k, r)$ rat Lonalizes $h$.

NOTATION 1. We denote by $F(X)$ the set of all fuzzy binary relations on $X$. we define $\Sigma=2^{x}$ and $C(X, \Sigma)$ denotes the sest of all rhojer functions $h$ on $x$. l.et $(R, \quad r) \varepsilon F(X)$. We use $(x, y) \varepsilon R$ and $x R:$ when $r(x, y) \neq 0$. L,t. $h \varepsilon\left(:\left(x, \sum\right)\right.$ be a choice function on $X$. Define $f(h)=\{(R, r) \varepsilon F(X):(R, r)$ rationalizes $h\}$.

DEFINITION 2. $h$ is said to be fuzzy transitive (total, reflexive) if there exists $(R, r)$ in $\varepsilon F(h)$ such that $(R, r)$ is transitw, (total, reflexive). (R, r) $F(X)$ is regular if $(R, r)$ is reflexile, total and transitive. $h$ is fuzzy regular if there exists $(R, r) \varepsilon F(h)$ such that $(R, r)$ is regular.

we shall prove the following theorem.

THEOREM 1. Every fuzzy rational cholce function is fuzzy transitive.

PROOF. Let $h$ be a fuzzy rational choice function on $x$. Then $F(h)$ is nonempty and let $(R, r) \varepsilon F(h)$. Then $h=h_{R}$. Suppose that $(R, r)$ is not transitive. Define $\{r\}=\{r(x, y) \neq 0: x, y \in X\}$ for $(R, r)$. We can find a positivie number $t_{0}=\frac{1}{n+k}$ such that $t_{0} \notin|r|$, where $k$ is a positive integer. We define a fuzzy relation $(S, s)$ as follows: If $r(x, y) \neq 0$, then we put $s(x, y)=r(x, y)$, and if $r(x, y)=0$ then we put $s(x, y)=t_{0}$. It is clear that $(S, s)$ is a transitive fuzzy relation on $X$. We show that $h_{R}=h_{s}$. To show this, we assume that $h_{R} \neq$ $h_{s}$. Then there exists a non-empty set $A$ such that $B=h_{R}(A) \neq h_{s}(A)=C$. We can assume that $c \in C$ and $a \notin B$. Then $(a, x) \in S$ for all $x \in A, s(a, x) \geq \frac{1}{|A|}>\frac{1}{n+k}$ $=t_{0}$, and hence $s(a, x) \neq t_{0}$. In view of $|r|$ and to $f|r|$, it is clear that $\mathbf{s}(a, x)=\mathbf{r}(a, x)$ for all $x \quad \varepsilon A$, and hence a $\varepsilon$. This contradicts a $k B$. A similar proof for $b \quad \varepsilon \quad B$ and $b \notin C$ brings a contradiction. Therefore $B=C$ and $h_{R}=h_{s}=h$. This proves Theorem 1 .

THEOREM 2. Every fuzzy rational choice function $h$ on $X$ is fuzzy total.

PROOF. Let $h$ be a fuzzy rational choice function on $x$. Then there exists $(R$, r) such that $h_{R}=h$. For $x, y \in X$ and $x \neq y$, it is clear that $h_{R}\{x, y\} \subseteq\{x, y\}$. Thus we have that either $r(x, y) \geqq \frac{1}{2}$ or $r(y, x) \geqq \frac{1}{2}$. Therefore $(R, r)$ is total. This proves Theorem 2 .

OOROLLARY 1. Every fuzzy rational choice function is regular. The proof follows from Theorems 1 and 2 .

3. A SEMIGROUP.

We begin with the following definition.

DEFINITION 3. Let $(R, r) \in F(X)$ be a fuzży relation. $(R, r)$ is completely total if $r(a, b) \neq 0$ and $r(b, a) \neq 0$ for $a l l a, b \in X$. A choice function $h$ is fuzzy completely total if there exists $(R, r) \quad \varepsilon F(X)$ such that $h_{R}=h$ and $(R, r)$ is completely total. $h$ is fuzzy completely regular if there exists ( $R, r)$ such that $h=h_{R}$ is fuzzy regular and fuzzy completely total.

We have considered a semigroup in [2] and [4]. We denote by $C R(X)$ the set of all completely regular fuzzy rational choice functions on $X$. By Theorem 4-(i)[2], we have that $h_{P} h_{Q} c_{-h} \cup Q$, $h_{p}$, he $\varepsilon C R(X)$. Thus we have the following theorem.

THEOREM 3. $C R(X)$ forms a semigroup under the binary operation defined by $h_{P} h_{Q}=h_{P} \cup Q$, $h_{P}, h_{Q} \varepsilon C R(X)$.

We note that if $h \varepsilon C R(X)$, then there exists $(P, p)$ such that $h=h_{P}$ and $(P, p)$ is regular and completely total.

PROOF. It is clear that the binary operation is associative. It is also clear that $P \cup Q=R(\operatorname{or}(R, r))$ is regular and completely total. Letting $P \quad U=$ 


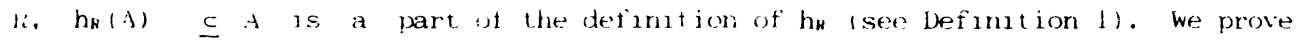
that $h_{R}(A)$ is non-empty when +1 - nom-empty. we assiume that $A \neq \emptyset$ and $|A|=$

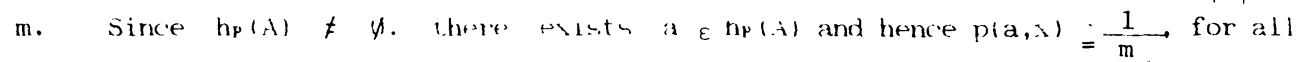

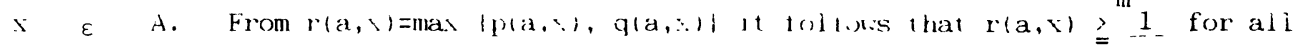

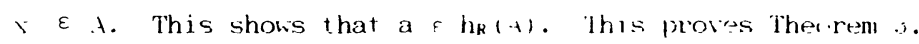

The following sample st to show that helhol, the romposite set function, is not a fuzzy ratsonal chesce eren though hp and ho are both fuzzy rational (holces on $X$.

FIAMPLE 1. Lot $h=\{a, h, c) . \quad$ Let $(k, r)=(r(a, a)=r(b, b)=r(c, c)=1$, $r(a, b)=r(a, c)=r(b, c)=\frac{1}{2}, \quad r(b, a)=r \cdot(c, a)=r(c, b) \quad$ and $(Q, q)=\left\{q(a, a)=q(b, b)=q(c, c)=1, q(b, a)=q(c, a)=q(c, b)=\frac{1}{2}, q(b, c)=\frac{1}{3}, q(a, b)=q(a, c)=\right.$ $\frac{1}{5}$. Then we can prove that there is not a fuzzy relation (P, p) such that $h_{P}=h_{R}\left(h_{Q}\right)$.

We list the followirg theorem.

THEOREM 4. Let. $(r, r)$ be a tuzzs I L Lat lor, on $X$. A necessary and sufficient condition for $h_{R}$ to be a rhoice furrtion ori $I$ is that for every non-empty subset $A$

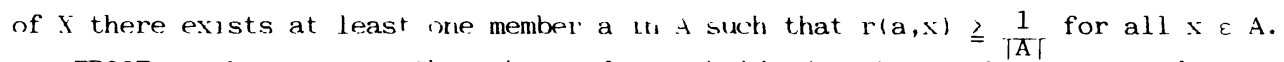

PROOF. We suppose that the rondition holds for $(k, r)$. Let $t \neq \emptyset$ and assume that there is a in $A$ such that $r(a, x) \doteq 1$ for all $\triangle E$ A. Then $A \subseteq R|A|(a)$ and $a \in h_{R}(A) . \quad h_{R}(A) \subseteq A$ ls a part of the definition of $h_{R}$. Thus hR is a chosce function on $\mathrm{X}$. Suppose $h_{\mathrm{k}}$ is a (husce on $\mathrm{A}$. Then for each $A \neq \emptyset$ there is a in $A$ such that a $\varepsilon$ hr $(t)$ trom whinh lif obtain that $r(a, x) \geq \frac{1}{|A|} \cdot \operatorname{This}$
proves Theorem $t$. 4. THE NUMBER OF ALL FUZZI RATIUNAL LHUICES UN $\{a, b, c$, d $\mid$. let $x$ be a set of $n$ elements. We denote the number of all tuzay rational choice functions on $\mathrm{I}$ by $h_{F}(x)(n)$. In $[2]$ we showed that $h_{F}(x)(3)=93$. In this section we announce that $h_{F}(x)(4)=57751$. WE shall prove this in a separate paper. A justification of $h_{F}(x)(4)=57751$ needs several pages.

\section{REFERENCES}

[1] K. J. Arrow, Social Choice and Individual Value (wiley, New York, 1963 ).

[2] Jin B. Kim, Fuzzy Rational (hoice Functions, FUZZY SETS AND SYSTEMS 10(1983), $37-43$.

[3] Jin B. Kim and Kern O. Kymn, Rational Choice and Gain Functions Derived From a Fuzzy Relation, EOONOMICS LETTERS 13)1983), 113-116.

[4] Jin B. Kim, Final Choice Functions, ECONOMICS LETTERS 14(1984), 143-148.

[5] Jin B. Kim, A Certain Matrix Semigroup, Mathematica Japonica 22(1978), 519-522.

[6] M. K. Richter, Rational Choice, in: J. S. Chipman, L. Hurwicz, M. K. Richter, and H. F. Sonnenschein, Eds., Preferences, Utility and Demand (Harcourt Brace Jovanovich, New York, 1971). 


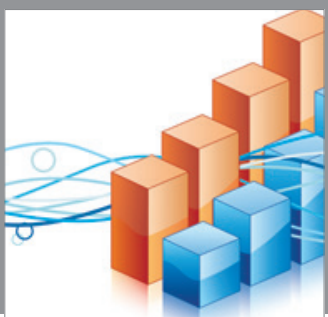

Advances in

Operations Research

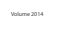

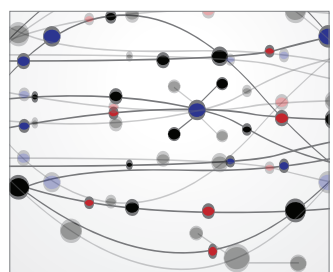

\section{The Scientific} World Journal
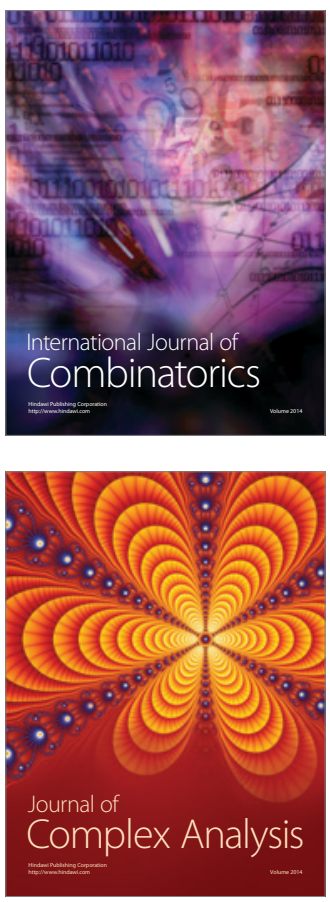

International Journal of

Mathematics and

Mathematical

Sciences
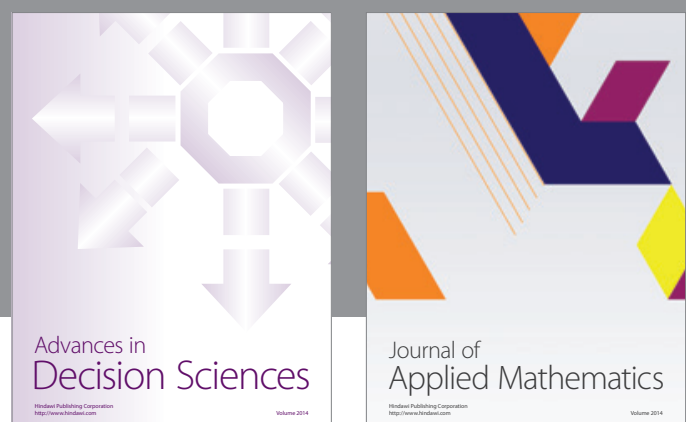

Journal of

Applied Mathematics
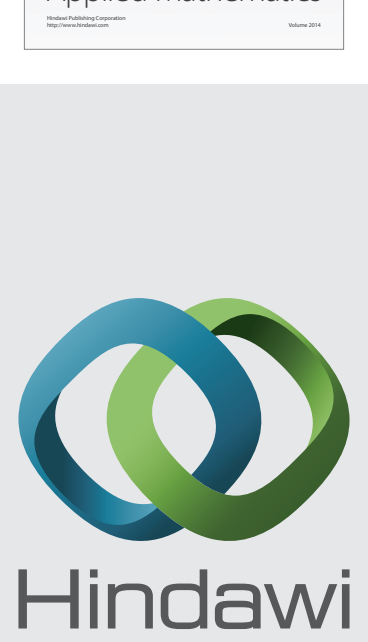

Submit your manuscripts at http://www.hindawi.com
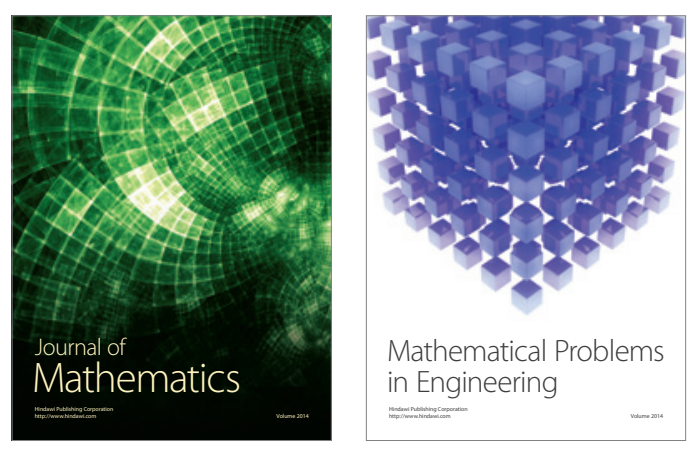

Mathematical Problems in Engineering
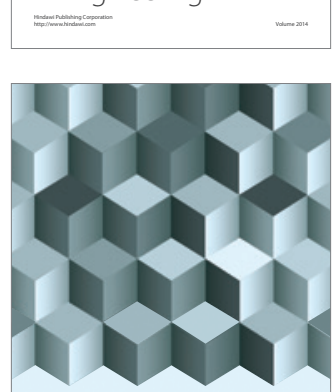

Journal of

Function Spaces
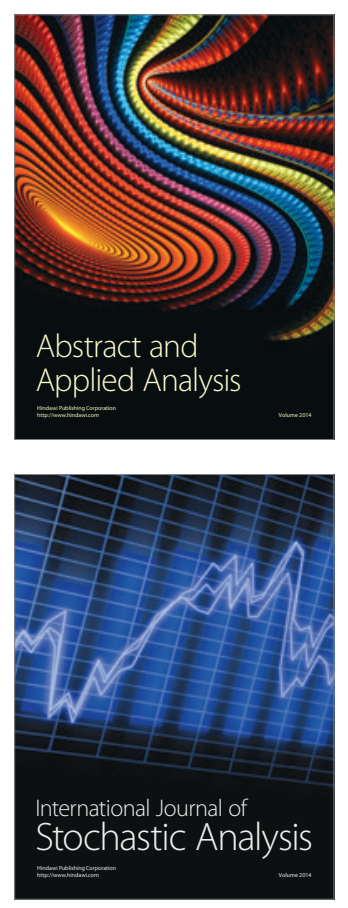

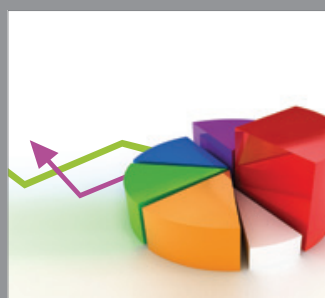

ournal of

Probability and Statistics

Promensencen
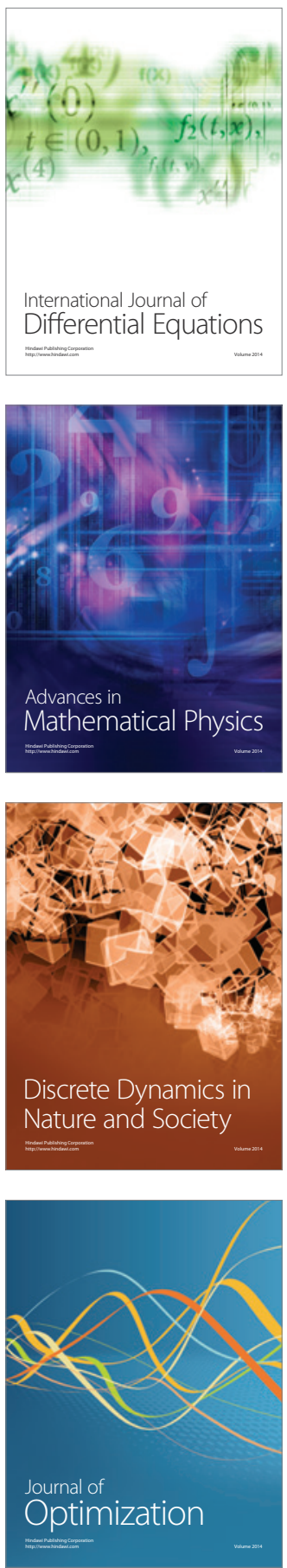